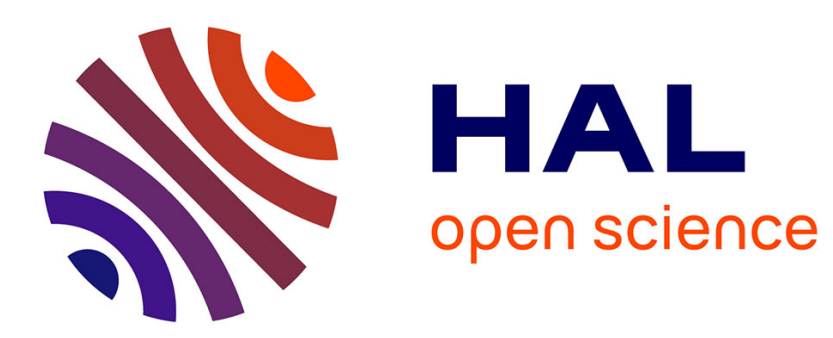

\title{
Texture Changes in the Plastic Deformation Zone Near the Fracture Surface of the Zr-1\% Nb Alloy Sheet
}

\author{
Yu. Perlovich, M. Isaenkova, V. Goltzev
}

\section{To cite this version:}

Yu. Perlovich, M. Isaenkova, V. Goltzev. Texture Changes in the Plastic Deformation Zone Near the Fracture Surface of the Zr-1\% Nb Alloy Sheet. Journal de Physique IV Proceedings, 1996, 06 (C6), pp.C6-335-C6-342. 10.1051/jp4:1996633 . jpa-00254461

\section{HAL Id: jpa-00254461 https://hal.science/jpa-00254461}

Submitted on 1 Jan 1996

HAL is a multi-disciplinary open access archive for the deposit and dissemination of scientific research documents, whether they are published or not. The documents may come from teaching and research institutions in France or abroad, or from public or private research centers.
L'archive ouverte pluridisciplinaire HAL, est destinée au dépôt et à la diffusion de documents scientifiques de niveau recherche, publiés ou non, émanant des établissements d'enseignement et de recherche français ou étrangers, des laboratoires publics ou privés. 


\title{
Texture Changes in the Plastic Deformation Zone Near the Fracture Surface of the $\mathrm{Zr}-1 \% \mathrm{Nb}$ Alloy Sheet
}

\author{
Yu. Perlovich, M. Isaenkova and V. Goltzev \\ Moscow Engineering Physics Institute, Kashirskoe shosse 31, Moscow 115409, Russia
}

\begin{abstract}
A wave of local plastic deformation moves in front of any crack and is responsible for strain hardening and rotation of crystalline lattice within the layer adjacent to the fracture surface. It was found by $\mathrm{X}$-xay diffractomctric study, that a directional reorientation of grains takes place near the fracture surface, leads to formation of the specific crystallographic textures and controls the further movement of the crack. The fracture anisotropy of the rolled zirconium alloy correlates with the character of observed texture changes in the plastic deformation zonc and can be predicted using the texture formation models by taking into account a variety of $\alpha-\mathrm{Zr}$ deformation micromechanisms. Alternative activation of prismatic slip or twinning depending on the tension direction determines both the thickness and the structure features of the plastic deformation zone near the fracture surface.
\end{abstract}

\section{INTRODUCTION}

Any plastic deformation of a metal material results in a crystallographic reorientation of its grains, even to the attainment of the final stable orientation, where lattice rotations caused by different active micromechanisms would be balanced out mutually. It is known that all deformation modes have their own characteristic textures with features predetermined by the active mechanisms. Regularities of deformation texture development are considered in the framework of the texture formation theory on the basis of models, connecting changes of the grain orientation with types of active micromechanisms [1-2]. The regular grain reorientation takes place not only under the homogeneous deformation over the whole sample, but also under a localized deformation in some microregion. A typical example of localized deformation is plastic deformation at the tip of the moving crack.

Substructure features of the layer adjacent to the fracture surface develop, when the plastic deformation zone at the tip of the moving crack goes through this layer. According to [3], at the crack tip the extending stresses prevail directed normally to the resultant fracture surface (Fig. 1). It was established by the method of dividing grids, that the form and the size of the plastic deformation field at the notch tip at the moment of crack start depend on the material treatment and the crack orientation. This field is always inhomogeneous and unequiaxed. Optic measurements have shown that in the case of plastic material within the zone up to $2 \mathrm{~mm}$ in thickness an extension degree was no less than $10-15 \%$ [4]. When taking into account an inhomogeneous distribution of plastic deformation at the crack tip, considerably higher deformation degree should be

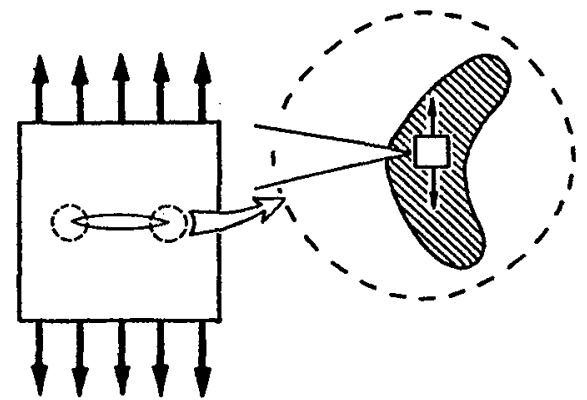

Figure 1: Prevailing stresses at the tip of the moving crack. 
expected in its immediate vicinity. Then the fracture occurs since the plasticity resource was exhausted by local attainment of the limiting extension degree. Such deformation degrees are accompanied necessarily by local formation of the extension texture with the axis perpendicular to the fracture surface.

In this work experimental verification of the specific texture changes near the fracture surfaces is presented in reference to the $\mathrm{Zr}-1 \% \mathrm{Nb}$ alloy consisting mainly of the $\alpha-\mathrm{Zr}$ phase with the hexagonal (HCP) crystalline lattice.

\section{METHOD OF INVESTIGATION: X-RAY TEXTURE ANALYSIS OF FRACTURE SURFACES}

The usual methods of X-ray diffractometric texture analysis [1,5] were modified to study texture features of fracture surfaces and adjacent layers. However, in order to apply X-ray methods for investigation of fracture surfaces, a stable wide-spread prejudice was to be overcome. It consists in steady following the traditional recommendation to use specimens with the smooth surface only. Roughness should be removed to avoid defocusing and to restrict X-ray diffraction from the surface layer with a distorted structure, as non-typical for the internal volume of the specimen. But in order to observe the structural changes connected with the crack movement, we should study just the fracture surface and adjoining layers, which have undergone an influence of plastic deformation at the crack tip. Hence, in the considered case the results of X-ray methods ought to be interpreted so as, in spite of instrumental errors because of surface roughness, the true information about substructure and texture features would be distinguished.

In the work [6] diffractometric recording of X-ray lines was used for layer-by-layer study of the strain hardening distribution nearby fracture surfaces of steel samples. It was shown that roughness of the studied fracture surfaces practically does not influence half-widths of registered X-ray lines. For fractures of different types a thickness of the adjacent zone with an increased distortion of crystalline lattice was determined. However, possibilities to analyse the processes in this zone on the basis of data about X-ray line half-widths are restricted because the dependence of half-widths on the deformation degree is not monotonic, a one-to-one correspondence between $\mathrm{X}$-ray line half-widths and active deformation micromechanisms is absent and the strain hardening distribution in grains with different orientations is inhomogeneous.

Usage of texture analysis is the next step on the way to adaptation of X-ray methods for investigation of fracture processes. Namely texture analysis allows to realize in full measure those important advantages, which X-ray methods have as compared with electron microscopy. Among them there are:

- the selective character of X-ray diffractometry, according to which any obtained information relates to the grains only with a definite orientation depending on the position of the studied sample with reference to the incident X-ray beam;

- the high statistical significance of the obtained data, conditioned by simultaneous X-ray reflection from the totality of separate grains with the same orientation, which are included in the textured matrix;

- the quantitative many-dimensional data form the reliable base for realistic models of fracture.

Besides, analysis of texture changes in the plastic deformation zone allows to identify the active deformation mechanisms and to predict the behaviour of the crack as it encounters grains with one or another concrete orientation.

The experimental procedures of texture measurement in the case of investigation of the fracture surface are the same as usually $[1,5]$. Some difference arises at the level of interpretation of the obtained data and will be considered below. By analysis of these data it is necessary to remember, that texture features of the layer near the fracture surface are caused by the wave of the inhomogeneous plastic deformation and therefore should be considered as a combined result of successive local deformations, which pass along the fracture surface in front of the moving crack. Thus, the study of local textures within microregions at different distances from the tip of the moving crack is substituted with the study of the final overall texture of the fracture surface, while the local deformations at different distances from the crack tip are characterized by the textures of successive layers.

The textures of investigated samples were characterized with inverse pole figures (IPF), which give the full crystallographic description of studied surfaces or cross-sections. IPF presents the distribution of the 
value $\mathrm{p}(\mathrm{hkl})$ in the elementary stereographic sector (in the case of material with a hexagonal crystalline lattice) or triangle (in the case of material with a cubic lattice), where $p(h k l)$ shows how many times the number of grains with the crystallographic plane $\{\mathrm{hkl}\}$ parallel to the studied surface is higher, than in the textureless sample. IPF's were measured and calculated by the modified X-ray method on the basis of diffractometric curves $\mathrm{I}_{i}\left(\alpha_{i}\right)$, describing changes of the registered intensity for several X-ray lines $(H K L)$, as the tilt angle $\alpha_{i}$ of the rotating sample increases [7]. Pole density $p(h k 1)$ for a point within IPF is proportional to the product $\Pi \mathrm{I}_{\mathrm{i}}$ of ordinates measured at the tilt angles $\alpha_{\mathrm{i}}$ equal to the angular distances of this point from the poles $(\mathrm{HKL})_{i}$. The treatment of experimental data and constructing of IPF's were carried out by use of the computer programs involving the procedures of data correction, interpolation and normalization.

For $\alpha-\mathrm{Zr}$ the X-ray lines $(0002),(10 \overline{1} 2),(10 \overline{1} 0)$ and $(11 \overline{2} 0)$ were registered, while the resulting IPF has a form of the stereographic sector with 219 rated points. The method provides a high sensitivity with respect to faint texture changes and allows to reveal minor variations of deformation processes within the investigated volume of material.

\section{STUDIED SAMPLES AND THEIR MECHANICAL PROPERTIES}

The anisotropy of fracture processes was studied as applied to the sheet of $\mathrm{Zr}-1 \% \mathrm{Nb}$ alloy. Samples for mechanical testing by tension were cut from the sheet $1 \mathrm{~mm}$ thick along rolling direction (RD) and along transverse direction (TD). Further these samples are labelled as RD- and TD-samples, while their studied cross-sections perpendicular to the tension axis are labelled as RD- and TD- sections respectively.

Samples of two types were prepared:

1) samples of size $185 \times 20 \mathrm{~mm}$ with the working part $130 \mathrm{~mm}$ long having a central notch $6.5 \mathrm{~mm}$ long; 2) plain samples of size $110 \times 8 \mathrm{~mm}$ with the working part $43 \mathrm{~mm}$ long.

Samples with a notch were tested at 293K; fracture parameters were measured by the method of dividing grids [4]. The grid with a cell size of $0.5 \times 0.5 \mathrm{~mm}$ was deposited near the notch tip. Start of the crack is accompanied by grid distortion, which is analyzed to determine the critical value of crack opening $\delta_{C}$, the stress of crack start $\sigma_{C}$ and the critical value of the integral $J_{C}[8]$. On stretching of notched samples up to breakage, the generated fracture surfaces were exposed to $X$-ray investigation. In order to increase the studied area of fracture surface, two or four identical tested samples were put together in the holder of the texture set. Polishing and etching of fracture surfaces were used only to a minimal degree.

In the case of $\mathrm{Zr}$-alloys, a thickness of the layer, within which an intensity of the incident X-ray beam reduces by one half, does not exceed several microns. Therefore the obtained IPFs give an averaged texture description of the layer at least up to $20 \mu \mathrm{m}$ in thickness.

To graduate the texture changes at the fracture surface of the notched samples, textures of the plain samples extended up to different deformation degrees were measured. It was assumed that the local deformation of material within the notched sample is equal to the homogeneous deformation of material with the same initial texture within the plain sample, if the registered changes of the chosen texture parameters in both cases are identical mutually.

The results of tensile testing of plain and notched samples are presented in Table 1.

Table 1 Mechanical properties and their anisotropy of the sheet $\mathrm{Zr}-1 \% \mathrm{Nb}$ alloy.

\begin{tabular}{|c|c|c|c|c|c|c|c|}
\hline \multirow{2}{*}{$\begin{array}{c}\text { Direction } \\
\text { of tension }\end{array}$} & \multicolumn{4}{|c|}{ Plain samples } & \multicolumn{4}{c|}{ Notched samples } \\
\cline { 2 - 8 } & $\sigma_{0.2}, \mathrm{MPa}$ & $\sigma_{\mathrm{b}}, \mathrm{MPa}$ & $\mathrm{K}_{\mathrm{Y}}$ & $\delta, \%$ & $\delta_{\mathrm{C}}, \mathrm{mm}$ & $\sigma_{\mathrm{Co}}, \mathrm{MPa}$ & $\mathrm{J}_{\mathrm{C}}, \mathrm{MPa} \mathrm{mm}$ \\
\hline $\mathrm{RD}$ & 211 & 362 & 0.72 & 25 & 0.82 & 383 & 24.7 \\
\hline $\mathrm{TD}$ & 273 & 335 & 0.23 & 21 & 0.87 & 390 & 30.1 \\
\hline $\begin{array}{c}\text { Anisotropy } \\
\text { coefficient }\end{array}$ & 0.77 & 1.08 & 3.13 & 1.2 & 0.98 & 0.94 & 0.82 \\
\hline
\end{tabular}


For each measured parameter the anisotropy coefficient $A$ is calculated as follows: $A(X)=X^{R D} / X^{T D}$ A relative variation of the anisotropy coefficient, which can be considered as physically meaningful one, is equal to $5 \%$.

Comparison of the stress-strain curves for plain RDand TD-samples (Fig.2) testifies about their sharp difference, consisting in the observed features of strain hardening. For its quantitative estimation the strain hardening indicator $\mathrm{K}_{\mathrm{Y}}$ is used, where

$$
\mathrm{K}_{\mathrm{Y}}=\left(\sigma_{0}-\sigma_{0,2}\right) / \sigma_{1,2}
$$

Values of $K_{Y}$ and the coefficient of strain hardening anisotropy are presented in Table 1 too. It is supposed that the analogous features of strain hardening take place in the plastic deformation zone at the tip of the moving crack.

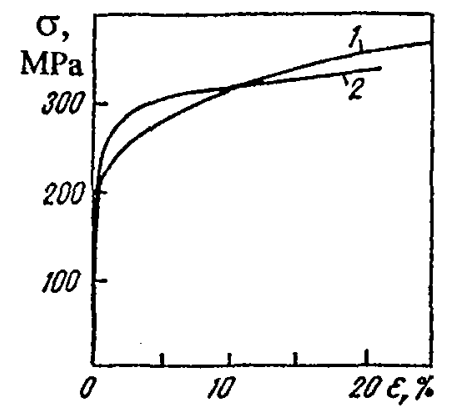

Jigure 2: Stress-strain curves for sheet samples of the $\mathrm{Zr}-1 \% \mathrm{Nb}$ alloy:

\section{RESULTS AND DISCUSSION}

1 - RD-tension; 2 - TD-tension.

Textures of RD- and TD-sections of plain samples both in the initial state and after tension as well as textures of the fracture surfaces are characterized by IPFs in Fig. 3. The initial textures of RD- and TD-
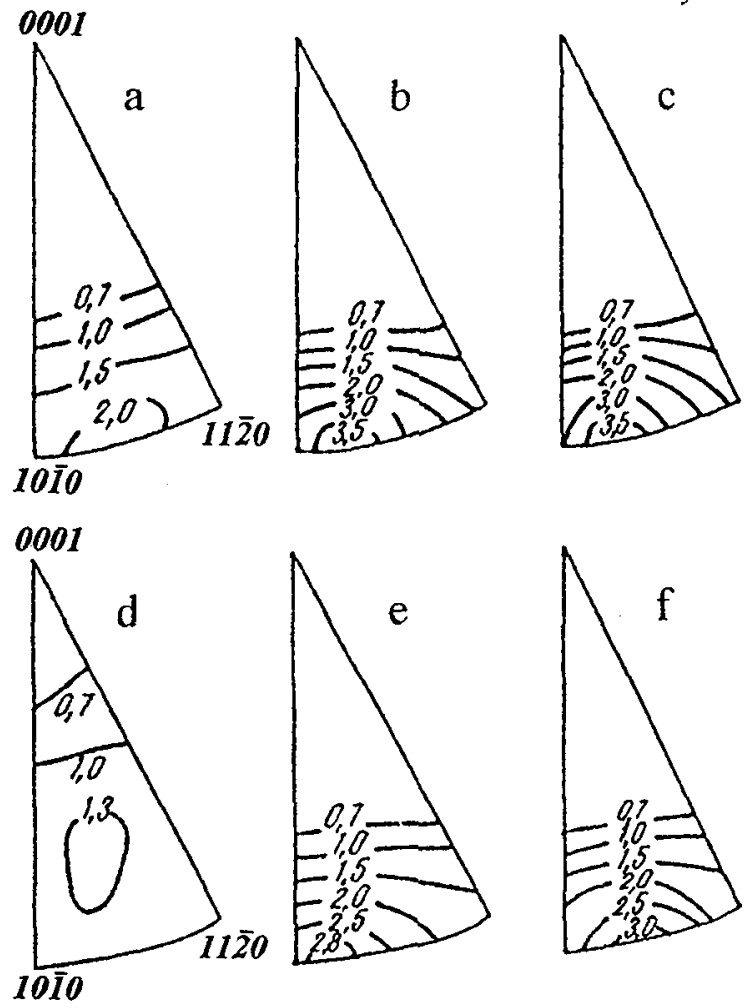

sections of the studied sheet differ sharply, what is typical for rolled metal materials with HCP crystalline lattice. IPF for RD-section (see Fig.3-a) contains the single texture maximum with a peak between poles $(10 \overline{1} 0)$ and $(11 \overline{2} 0)$, testifying that the original sheet was recrystallized partially [2]. IPF for TD-section also contains only one texture maximum, but it is severely scattered and shifted to center of the stereographic sector (Fig.3-d).

As to textures of the fracture surfaces, it can be seen in Fig. 3-c, $f$ that to a first approximation they are similar and fall in the same type with the initial texture of RD-section, though being significantly more perfect. An essential grain reorientation near the fracture surface is especially evident in the case of the TD-sample, where it is connected with principle changes of the initial texture. In order to demonstrate that the registered texture changes near the fracture surface of notched samples are caused by local tensile deformation, these texture changes were compared with changes observed for plain samples, experienced tension up to different deformation degrees.

According to [9], the tension texture of $\alpha-\mathrm{Zr}$ is characterized by predominant alignment of crystallographic axes $<1010\rangle$ along RD. Hence, as a result of tension the texture maxima in IPF's for the section perpendicular to the tension axis would be shifted to the pole (1010), while their scattering would decrease. This prediction is supported in full measure by texture analysis of
Figure 3: Texture changes in $\mathrm{RD}-(\mathrm{a}, \mathrm{b}, \mathrm{c})$ and TD-sections (d, $e, f$ ) of the sheet $\mathrm{Zr}-1 \% \mathrm{Nb}$ alloy owing to tension of plain samples $(b, e)$ and fracture of notched samples $(c, f)$ :

a - RD-section, initial state; d - TD-section, initial state;

b - RD-tension by $22 \%$;

c - fracture surface of RD-sample; e - TD-tension by $10 \%$;

$f$ - fracture surface of TD-sample. 
of the corresponding sections for stretched plain samples. Thus, in IPF for RD-section the texture maximum top, which was offset initially by $15^{\circ}$ from the pole (1010), in consequence of RD-tension by $22 \%$ neared it by $7^{\circ}$, while an angular width of the texture maximum at a half of its height decreased from $40^{\circ}$ down to $34^{\circ}$ (Fig.3-b). In the case of TD-tension at the very first deformation stage the texture maximum moves abruptly to the external boundary of the stereographic sector, and then, more slowly already, moves to the pole (1010); after tension of TD-sample only by $10 \%$ the top of the texture maximum in IPF approached the pole (1010) at an angular distance of $5^{\circ}$ already, while its half-width decreased from $64^{\circ}$ down to $30^{\circ}$ (see Fig.3-e), testifying about the higher reorientation speed of $\alpha-\mathrm{Zr}$ grains, than in $\mathrm{RD}$-sample.

It follows from the works on plastic deformation mechanisms and texture formation in $\alpha-Z \mathrm{r}[2,10]$, that the grain reorientation by tension of RD-sample is caused by prismatic slip, whereas by tension of TD-sample prismatic slip is preceded by intense twinning, resulting in an abrupt shift of the texture maximum from its initial position.

Comparison of the obtained IPFs testifies quite unambiguously, that grain reorientations at the tip of the moving crack are similar to those in the corresponding sections of stretched plain samples. Presence of the notch proves to have no influence on the anisotropic behaviour of material. On the basis of texture changes in the stretched plain samples the graduation of plastic deformation near the fracture surface of the notched samples was carried out. The quantity $q=p(10 \overline{1} 0) / p(11 \overline{2} 0)$ was chosen as a characteristic parameter of IPF, increasing as the texture of tension develops in the sample. Using the curve $q(\varepsilon)$, constructed by texture study of the stretched plain samples, the deformation degrees near the fracture surface of the notched samples were estimated. Fig.4 illustrates this procedure. The obtained value characterizes an averaged deformation degree for the layer up to $20 \mu \mathrm{m}$ in thickness, participating in X-ray diffraction by IPF measurement.

It was determined that near the fracture surface in $\mathrm{RD}$-samples the material experiences the tensile deformation by $15-19 \%$, while in TD-samples - by 3-5\%. Because of the inhomogeneous distribution of plastic deformation near the fracture surface, the

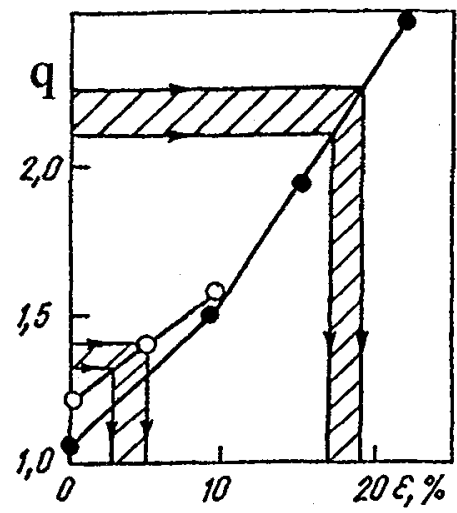

Figure 4: Estimation of the deformation degree at the fracture surface by use of the texture parameter $\mathrm{q}=\mathrm{p}(1010) / \mathrm{p}(1120)$ : o- RD-tension, o-TD-tension.

real local deformation degree for the layer of several microns in thickness, adjoining immediately to this surface, is essentially higher than the above-indicated values. The fracture takes place, when the plasticity resource of the localized deformation layer is exhausted,- hence, both in RD- and TD-samples in the immediate vicinity of the notch tip the greatest possible deformation degree is attained. Then the results, obtained by the graduation curve $q(\varepsilon)$, characterize a thickness of the localized deformation layer near the fracture surface rather than the maximal deformation degrees of $\alpha-\mathrm{Zr}$ grains in this layer. The lower is the deformation degree near the fracture surface according to the curve $q(\varepsilon)$, the thinner is the studied layer of localized deformation at the crack tip. That is to say that in RD-samples this layer is significantly thicker, than in TD-samples.

Thus, by testing of notched samples the same processes of grain reorientation and texture changing develop, as by tension of plain samples, with the only difference that in notched samples these processes are localized near the fracture surface.

Texture changes, observed in $\alpha-\mathrm{Zr}$ near the fracture surface, can be used as a starting point for analysis of the anisotropic resistance to fracture with reference to the studied sheet alloy $\mathrm{Zr}-1 \% \mathrm{Nb}$. Reorientation of grains at the tip of the moving crack follows from their natural tendency to attain an orientation, which would be stable relative to the predominant tensile deformation. Stability of such final grain orientations is conditioned by mutual equilibrium of active deformation micromechanisms, resulting in opposite lattice rotations. As a result of slip, caused by tensile deformation, the grain lattice rotates in such a way, that the direction of slip in the active system tends to approach the axis of tension [1]. As the lattice rotates, shear 
stresses both in active and potential slip systems change, and sooner or later additional systems would be activated, having an effect on the resulting lattice rotation. On the sufficiently high deformation degree the grain gets to a final stable orientation, where rotations of its lattice due to different slip systems are mutually compensated. In $\alpha-\mathrm{Zr}$ the stability of the tension axis final orientation $\langle 10 \overline{1} 0\rangle$ is provided by balanced action of two prismatic slip systems.

The trajectories of grain reorientation under the action of tension are strictly regular and depend on the suquence of activated slip and twinning systems, which is rather well-known with reference to $\alpha-\mathrm{Zr}$ [1]-12]. From the standpoint of fracture resistance the special significance has the influence of lattice reorientation at the crack tip on the strain hardening of grains in connection with its orientational dependence. The minimal strain hardening takes place in the grains deformed on account of slip by a single system. When active slip systems grow in number, strain hardening of the grain increases. Besides, the effects of texture hardening and dishardening are known, involving respectively a decrease or an increase of shear stresses in the active system as the grain changes its orientation [3]. In particular, the distribution of the Schmid factor for prismatic slip systems in HCP-lattice is such that its maximum $(0.50)$ is situated at the external boundary of the stereographic sector at the same distance of $15^{\circ}$ from poles (1120) and $(10 \overline{1} 0)$, whereas by approach of the tensile axis to its final stable position at the pole (10ĩ) the Schmid factor in the active prismatic slip system decreases down to 0.44 [9]. Therefore, independently of the dislocation hardening, tension of the RD-sample should be accompanied by the hardening, having the pure geometrical nature and depending on the orientation of the slip plane relative to the axis of tension.

Upon tension of the RD-sample the prismatic slip results in gradual changing of grain orientations, while the strain hardening is overcome owing to an additional loading. In the TD-sample only few grains had initial orientations favourable for preferable activation of the prismatic slip. At the same time in many grains owing to their orientation the twinning activates first of all, being testified by the abrupt redistribution of pole density in IPF. In $\alpha-\mathrm{Zr}$ the critical shear stress for twinning is higher, than for prismatic slip [12], - therefore the conventional yield stress $\sigma_{0.2}$ for the TD-sample, which at the initial stage of tension deforms predominantly by twinning, appears to be higher than for RD-sample, deforming by prismatic slip.

Mutual intersection of the stress-strain curves for RD- and TD-samples (see Fig.2) testifies that the further deformation of the TD-sample on prismatic slip activation is connected with a weaker hardening than in the case of $\mathrm{RD}$-sample. The weaker hardening of the TD-sample is conditioned by the following circumstances:

- an external force necessary for activation and maintenance of prismatic slip in the grains, which previously have experienced twinning and reoriented favourably, is lesser than that required before for activation of twinning, so that the initially attained stress provides deformation development at the further stage of predominant slip as well;

- activation of the prismatic slip in the majority of grains within the TD-sample takes place, when the tensile axis, either owing to the initial texture or in consequence of preliminary twinning, falls at the periphery of maximum in the Schmid factor distribution on the side of the pole (10 $\overline{1} 0)$ [9], so that the following rotation to this pole does not involve the geometric (texture) hardening.

The process of loading was considered by taking into account that the tip of the notch is a stress concentrator. As the load increases, deformation activates first of all in the layer adjoining immediately to the notch, where the maximal stress is attained. Only when the strain hardening of material within this layer begins to increase, deformation extends gradually for the more distant layers. Obviously, the less is the critical shear stress for the active deformation mechanism, the further from the notch tip extends the zone of localized plastic deformation. In particular, the layer-by-layer distribution of deformation degree near the notch tip in RD-samples depends on the strain hardening of $\alpha-\mathrm{Zr}$ grains in the process of prismatic slip.

The presented experimental data testify that by testing of TD-samples the load increases until activation of the twinning within some thin layer near the notch tip. In consequence of the resulting abrupt reorientation and the corresponding increase of shear stresses in the prismatic systems, the twinned grains obtain at once the ability for the following deformation by means of prismatic slip. Activation and maintenance of the latter mechanism requires for a lesser load than the one achieved before, and this circumstance prevents the extending of twinning for other layers. Only in the event that the strain 
hardening owing to prismatic slip in the first layer exceeds the difference of critical shear stresses for twinning and prismatic slip, the following load rise up to the level, providing activation of twinning in the next layer, appears to be possible.

Thus, extention of the plastic deformation zone from the notch tip inwards the sample takes place in different manners in the cases of RD- and TD-samples: as the deformation degree of material near the notch tip increases, the plastic deformation zone in the RD-samples widens continuously, whereas in the TD-sample it widens discretely, remaining unaltered in the course of some loading stages. While the plastic deformation zone in the RD-sample has not a distinct boundary, such a zone in the TD-sample is restricted by an abrupt border. Outside of this border the twinning was not activated and, as a result, its crossing is accompanied by the jump-like change of the local texture (Fig.5). Therefore the layer of localized deformation in the $\mathrm{RD}$-sample is thicker than in the TD-sample. (The denser is hatching in Fig.5, the higher is the degree of local deformation by slip.)

By analogy with notched samples, plain TD-samples under tension show a less homogeneous deformation than RD-samples. Even in the absence of stress concentrators like a notch tip, at some areas of the TD-sample the twinning takes place earlier than at others owing to accidental factors. This inevitable fact proves to be a sufficient prerequisite for a slight localization of deformation development by means of the subsequent prismatic slip. Inhomogeneous deformation and the resulting texture inhomogeneity tend to increase the density of high-angle boundaries and, by doing so, promote additional localization of deformation, rising the probability of fracture. It is natural that exhaustion of the plasticity resource under conditions of localized deformation occurs at a lesser averaged stress and at a lesser deformation degree of the

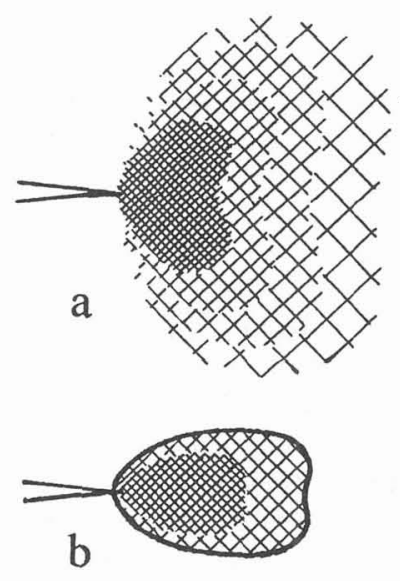

Figure 5: A schematic sketch of the plastic deformation zone at the tip of the moving crack for the cases of RD- (a) and TD-tension (b).

crack tip, conditions of homogeneous deformation. Namely therefore both the tensile strength $\sigma_{b}$ and the relative elongation for the TD-sample are found to be less than for the $\mathrm{RD}$-sample (see Table 1).

When considering the results of mechanical testing in Table 1, the anisotropy of fracture resistance for the studied sheet does not correspond to the anisotropy of properties measured for plain samples. Though the tensile strength $\sigma_{b}$ for the RD-sample is higher than for the TD-sample, the crack propagation in it requires lesser energy expenditures, being testified by comparison of $\mathrm{J}_{\mathrm{C}}$-integral values for $\mathrm{RD}$ - and TD-samples. The work of deformation, which precedes fracture, is proportional to an area under the "stress-strain" curve (Fig.2). Since $\sigma_{0.2}{ }^{\mathrm{TD}}>\sigma_{0.2}{ }^{\mathrm{RD}}$ and the registered anisotropy of coefficient $\mathrm{J}_{\mathrm{C}}$-integral is connected with the fact that within the observed zone near the notch tip the local deformations up to $15-20 \%$ prevail, an area under the TD-curve is still more than under the RD-curve. At the same time, the zone of high local deformations, close to the limiting one, is so small, that has no essential effect on general energy expenditures.

It is evident also, that by the crack start moment the size of the plastic deformation zone in the TD-sample, in spite of the strong localization of deformation, is comparable with the size of the analogous zone in the RD-sample. This fact occurs to be possible owing to significant scattering of the initial texture in the TD-sample, such that already at the early stage of tension the prismatic slip is activated in a relatively small fraction of grains. 


\section{CONCLUSIONS}

1. Crystallographic reorientation of grains at the tip of the moving crack was revealed for the first time by the example of the sheet $\mathrm{Zr}-1 \% \mathrm{Nb}$ alloy.

2. Presence of the notch does not change the anisotropic behaviour of the studied material.

3. The effects observed by use of X-ray diffractometry near the fracture surface of notched samples were compared with the effects in the body of analogous plain samples deformed by tension. The estimates show that the averaged tensile deformation of material within the layer up to $20 \mu \mathrm{m}$ in thickness near the fracture surface comprises $15-20 \%$ for the RD-sample and 3-5\% for the TD-sample.

4. The fracture resistance anisotropy and development of the plastic deformation zone at the crack tip are determined by the activated mechanisms of $\alpha-\mathrm{Zr}$ plastic deformation, whose characteristic feature is the strong dependence on the crystallographic orientation of grains.

\section{References}

[1] Wasserman G., Greven J., Texturen metallischer Werkstoffe (Springer -Verlag, Berlin / Göttingen / Heidelberg, 1962) $808 \mathrm{~S}$.

[2] Vishnyakov Ya., Babareko A., Vladimirov S. and Egis I., The theory of texture formation in metals and alloys (Nauka, Moscow, 1979) $344 \mathrm{p}$.

[3] Honeycombe R.W.K., The plastic deformation of metals (Edward Arnold LTD, 1968) 477 p.

[4] Goltzev V., Zelenskiy A., Kudryavzev O. and Matvienko Yu., "The zones of localized plastic deformation in preliminary deformed sheet plastic materials",An investigation into strength of materials and constructions of atomic technique (Energoatomizdat, Moscow, 1984) pp.68-73.

[5] Borodkina M., Spektor E., X-rays texture analysis of metals and alloys (Metallurgiya, Moscow, 1982) $272 p$.

[6] Klevtsov V., Zavodskaya Laboratoriya 3 (1992) 81-89.

[7] Perlovich Ju., Struev S. and Kapliy S., "A new precise X-ray method for building of inverse pole figures", 10th International Conference on Textures of Materials. Abstracts (TU Clausthal, Deutsche Gesellschaft für Materialkunde) p. 137.

[8] Bruchmechanische Werkstoffcharaktersierung, H. Blumenauer Hrsg. (Deutscher Verlag für Grundstoffindustrie, Leipzig, 1991) $176 \mathrm{~S}$.

[9] Calnan E.A., Clews C.J.B., Phil.Mag. 42 (1951) 919-931.

[10] Isaenkova M., Perlovich Yu., Fizika metallov i metallovedenie 64 (1987) 107-1 12.

[11] Douglass D.L., The metallurgy of zirconium ( International Atomic Energy Agency, Vienna, 1971) $465 \mathrm{p}$.

[12] Tenckhoff E., Verformungsmechanismen Textur und Anisotropie in Zirkonium und Zircaloy. Materialkundlich-Technische Reihe, 5 (Gebrüder Borntraeger, Berlin-Stuttgart, 1980) 79 S. 